\title{
Gas Measurement
}

National Cancer Institute

\section{Source}

National Cancer Institute. Gas Measurement. NCI Thesaurus. Code C82624.

A measurement of the gas in a sample. 